\title{
Male germ cell-specific protein Trs4 binds to multiple proteins
}

\author{
Yu-Qiang Shi ${ }^{\text {a,b,1 }}$, Yin-Chuan $\mathrm{Li}^{\mathrm{a}}{ }^{\mathrm{a} 1}$, Xiao-Qian $\mathrm{Hu}^{\mathrm{a}}$, Tao Liu ${ }^{\mathrm{a}}$, Shang-Ying Liao ${ }^{\mathrm{a}}$, Jian Guo ${ }^{\text {a }}$, Lin Huang ${ }^{\mathrm{a}}$, \\ Zhao-Yuan Hu ${ }^{a}$, Alan Y.B. Tang ${ }^{c}$, Kai-Fai Lee ${ }^{c}$, William S.B. Yeung ${ }^{c}$, Chun-Sheng Han ${ }^{\text {a,* }}$, Yi-Xun Liu ${ }^{\text {a,* }}$ \\ a State Key Laboratory of Reproductive Biology, Institute of Zoology, Chinese Academy of Sciences, Beijing 100101, PR China \\ ${ }^{\mathrm{b}}$ College of Bio-Engineering, Weifang University, Weifang, Shandong Province 261061, PR China \\ ${ }^{\mathrm{C}}$ Department of Obstetrics and Gynaecology, Li Ka Shing Faculty of Medicine, The University of Hong Kong, Pokfulam, Hong Kong, PR China
}

\section{A R T I C L E I N F O}

\section{Article history:}

Received 31 July 2009

Available online $\mathrm{xxxx}$

\section{Keywords:}

Trs4

IQ calmodulin-binding motif

Testis

Cryptorchidism

Spermatogenesis

\begin{abstract}
A B S T R A C T
Temperature-related sequence 4 ( $\operatorname{Trs} 4$ ) has been identified as a testis-specific gene with expression sensitive to the abdominal temperature changes induced by artificial cryptorchidism. In murine testes, Trs 4 mRNA was detected in round spermatids and its protein was localized mainly in the elongating spermatids as well as in the acrosomes and tails of mature spermatozoa. Using a yeast two-hybrid screening system, we identified Rshl-2, Gstmu1, and Ddc8 as putative binding partners of the Trs4 protein in mouse testes. Their interactions were confirmed by in vivo and in vitro binding assays. Further studies demonstrated that $D d c 8$, a newly identified gene with unknown functions, displayed a similar expression pattern with Trs4 in mouse testes. In particular, Trs4, Ddc8, and Rshl-2 proteins were co-localized to the tails of mature spermatozoa. These results suggested that Trs 4 might be involved in diverse processes of spermiogenesis and/or fertilization through interactions with its multiple binding partners.
\end{abstract}

(c) 2009 Published by Elsevier Inc.

\section{Introduction}

Spermatogenesis is a complex process involving mitosis in spermatogonia, meiosis in spermatocytes and dramatic morphological changes to spermatids leading to the production of ciliated mature spermatozoa. This is a highly regulated process in which many testis-specific genes are involved [1]. It is estimated that about $4 \%$ of the genes in the mouse genome are testis-specific: most are transcribed in round spermatids but are not translated until the proteins are needed in later stages [2]. A number of highly specialized strategies for gene regulation are adopted during spermatogenesis, including a unique chromatin reorganization program, the use of distinct promoter elements and specific transcription factors [3]. Defective spermatogenesis is a major cause of human male infertility [4], so elucidating the functions of these testis-specific genes will not only help in understanding the mechanism of spermatogenesis, but might also allow the development of novel treatments for male infertility [2].

Spermatocytes and spermatids are very sensitive to many internal and external stresses. For example, they undergo apoptosis in response to hyperthermia [5,6]. Spermatogenesis occurs in the testes at temperature $4-5{ }^{\circ} \mathrm{C}$ lower than the body core temperature in most mammals. Patients with testes in the abdomen cavity (crypt-

\footnotetext{
* Corresponding authors. Fax: +86 1064807105 (C.-S. Han), +86 1064807038 (Y.X. Liu).

E-mail addresses: hancs@ioz.ac.cn (C.-S. Han), liuyx@ioz.ac.cn (Y.-X. Liu).

1 These authors contributed equally to this work.
}

orchidism) suffer from spermatogenetic impairments, which can be restored by bringing the cryptorchid testes surgically back to the scrotum [7]. We and others have used animal models to study the effect of artificial cryptorchidism or heat shock on spermatogenesis $[8,9]$ and two novel heat-sensitive genes from rat spermatids, T6-441 and Afaf were identified [10,11]. Using a cryptorchid rat model, we have isolated an expressed sequence tag (EST) in rat testes, named $\operatorname{Trs} 4$ (temperature-related sequence 4) [12]. Here we report the cloning and expression pattern of Trs 4 and its interaction with proteins including Rshl-2, Ddc8, and Gstmu1 in mouse testes. We hypothesize that Trs4 might be involved in diverse processes of spermiogenesis and/or fertilization through interactions with its multiple binding partners.

\section{Methods}

Animal experiments. Sprage-Dawley (SD) rats and CD-1 mice were obtained from the Experimental Animal Center, Chinese Academy of Sciences. All animals were treated in accordance with the NIH Guide for the Care and Use of Laboratory Animals and all protocols were approved by the Committee of Animal Care and Use of the Institute of Zoology, Chinese Academy of Sciences. The procedure for artificial cryptorchidism was performed as described before [12].

Reagents. If not stated otherwise, all reagents for cell culture were purchased from Invitrogen (Carlsbad, CA) and Sigma-Aldrich (St. Louis, MO). The Matchmaker library construction and screening 
kit, SMART RACE cDNA Amplification Kit and Matchmaker Co-IP kit were purchased from Clontech (BD Biosciences. San Jose, CA). Antigreen fluorescent protein (GFP) monoclonal antibody was purchased from Santa Cruz Biotechnology (Santa Cruz, CA). AntiFlag monoclonal antibody was purchased from Sigma-Aldrich.

Rapid amplification of $5^{\prime}$ - and $3^{\prime}-c D N A$ ends. Total RNAs from adult SD rat and CD-1 mice testes were extracted to synthesize complementary DNA (cDNA). Rapid amplification of $5^{\prime}$ and $3^{\prime}$ complementary DNA ends (RACE) was performed using SMART RACE cDNA Amplification Kits. Domain and motif analysis followed the annotations of the NCBI Conserved Domain Database (http:// www.ncbi.nlm.nih.gov/Structure/cdd/cdd.shtml). Multiple sequence alignment was performed using the EBI ClustalW service (http://www.ebi.ac.uk/Tools/webservices/services/clustalw).

Recombinant protein expression and polyclonal antibody production. The mouse Trs4 cDNA fragment (amino acids 31-101) and mouse Ddc8 cDNA fragment (amino acids 182-282) were subcloned in-frame into the pET21b(+) vector with the C-terminal tagged with His6 peptide. His-tagged fusion protein was then expressed in Escherichia coli strain BL21 and purified using a HisTrap FF column (Amersham Pharmacia. Piscataway, NJ). The antibodies were produced and purified as described [11].

Northern blotting, in situ hybridization, and reverse transcriptionpolymerase chain reaction ( $R T-P C R)$. Total RNAs from multiple adult rat tissues were extracted for Northern blotting and RT-PCR analysis. The primer pairs, $5^{\prime}$-TTG CTT CCA TCG GGA GAC ATA G-3' and 5'-CGT GTT TAT CCC ACC TGA CCA T-3' were used to synthesize probes for Northern blotting and in situ hybridization as described [10]. The same primers were also used to detect mRNA expression in rat testes by RT-PCR. Sertoli and Leydig cells from adult mice were prepared by two-step enzymatic digestion and cultured as described [13]. Total RNAs from mouse testes at different stages of development, the cultured primary testicular cells and the GC2-spd spermatocyte cell lines were extracted for RT-PCR analysis. The primer pairs $5^{\prime}$-aaa ttg agg ttg gag tgg ga-3' and 5'-ATA CGA GCA GCG GAT CTA TG-3', 5'-GAC CAC CTT AGG ACA AGA TTG- ${ }^{\prime}$ and 5'-TCC TGC CAC TCT GCC TTT AAC- $3^{\prime}$, were used to detect Trs 4 and $D d c 8$ mRNA expression in mouse testes by RT-PCR, respectively.

Confocal fluorescence and immunohistochemistry. The whole coding sequence of mouse Trs 4 cDNA was cloned in-frame into pEGFPN1 to construct a Trs4-GFP recombinant plasmid. The Trs4-GFP-fusion protein was expressed in HeLa cells using Lipofectamine 2000 transfection reagent. Anti-Trs4 antibody (1:500) and TRITC-conjugated anti-rabbit secondary antibody $(1: 200)$ were used for indirect immunostaining. To investigate the localization of Trs4 and Ddc8 proteins in mature spermatozoa, mouse cauda epididymidis was isolated and incubated in DMEM medium. The dissociated sperm were collected and smeared on glass slides. After fixing and blocking, the slides were incubated with anti-Trs4 or anticontrol). Subsequently, the slides were incubated with FITC-conjugated anti-rabbit secondary antibody (1:200). The nuclei were counterstained with 4',6-diamidino-2-phenylindol (DAPI) and the images were captured using a laser confocal microscope (Zeiss). Immunohistochemistry was performed as described [11].

Yeast two-hybrid screening. Yeast two-hybrid screening was performed using the Matchmaker library construction and screening kit with some modifications [14]. Two bait plasmids, T1 encoding the $N$-terminal 407 amino acids of mouse Trs $4(22-428)$ and T2 encoding the $C$-terminal 500 amino acids of mouse Trs4 (281780) were constructed.

Coimmunoprecipitation. To confirm their interactions in vivo, cDNA fragments of mouse Trs 4 bait plasmid T2 and its interacting proteins were subcloned into pFlag-CMV4 and pEGFP-N1 vectors to construct Flag and GFP-tagged plasmids, respectively. The Flag-Trs4 was overexpressed in HEK293T cells with each of its Ddc8 antibodies (1:400) or preimmune rabbit serum (negative
GFP-tagged interacting proteins using Lipofectamine 2000. Total protein lysates of the cells were incubated with anti-GFP antibody or rabbit immunoglobulin ( $\operatorname{IgG}$ ) for $2 \mathrm{~h}$ at $4{ }^{\circ} \mathrm{C}$, followed by incubation with protein A coated agarose beads overnight at $4{ }^{\circ} \mathrm{C}$. The agarose beads and captured protein complexes were washed six times and suspended in SDS sample buffer for immunoblotting with anti-GFP (1:1000) or anti-Flag (1:2000). In vitro binding assays were performed using the TNT $^{\circledR}$ Quick Coupled Transcription/Translation Systems (Promega, Madison, WI) and Matchmaker Co-IP kit as described before [14].

Western blot analysis. Equal amount of protein $(50-100 \mu \mathrm{g}$ total protein/lane) were loaded and separated by SDS-PAGE. After the proteins were transferred to polyvinylidene fluoride (PVDF) membranes, the membranes were blocked and incubated with primary antibodies, followed by incubation with HRP-conjugated secondary antibody. Proteins were visualized by Enhanced Chemiluminescence kit (Pierce. Rockford, IL).

Statistics. Band intensities were analyzed and normalized with internal controls. Values are represented as means \pm SEM of three separate experiments. Statistical analysis was performed using SPSS (version 13.0; SPSS Inc., Chicago, IL) and one-way ANOVA was used to analyze the data in different groups; $P<0.05$ was assumed significant.

\section{Results}

Cloning and sequence analysis of Trs4

The sequence of rat Trs4 (GenBank Accession No.: DQ132434) was obtained by RACE and sequence analysis showed that rat Trs4 has a full length of 2370 nucleotide acids encoding a putative protein of 790 amino acids. Multiple sequence alignment indicated that the proteins are highly conserved across species. Importantly, a putative IQ calmodulin-binding motif and an ubiquitin-like structure (DUF2021 in the PFAM database; http://pfam.sanger.ac.uk/) were found in all the species tested (Supplementary Fig. 1).

\section{Expression profile of Trs4 mRNA in murine testes}

Trs4 was specifically expressed in the rat testis and no expression was found in other tissues including the ovary, kidney, heart, liver, spleen, lung, brain and stomach by RT-PCR (Fig. 1A). This expression pattern was further confirmed by Northern blotting in rat tissues (Fig. 1B). A single transcript of about 3000 nucleotides was visualized on the blot, consistent with the result of RACE analysis. The developmental profiles of Trs 4 transcripts in testes were examined by Northern blotting in rats (Fig. 1C) and RT-PCR in mice (Fig. 1D), respectively. Trs 4 mRNA was first detected at 28 days post partum (dpp) in rats and $21 \mathrm{dpp}$ in mice, at the time when round spermatids are produced in the first wave of spermatogenesis [15]. No expression was found in Sertoli cells, Leydig cells or GC2-spd cells cultured in vitro (Fig. 1D). In situ hybridization results confirmed that Trs4 mRNA was localized in round spermatids of stages VII-VIII seminiferous tubules in mouse testes (Fig. 1E). Furthermore, the Trs 4 transcript in rat testis was found to be sensitive to intra-abdominal temperature and was deceased significantly after artificial cryptorchidism (Supplementary Fig. 2), which is consistent with our previous report [12].

Trs4 protein is localized in early elongating spermatids and mature spermatozoa

HeLa cells were transfected with Trs4-GFP fusion plasmid for detecting specificity of Trs 4 antibody and its subcellular localiza- 
A

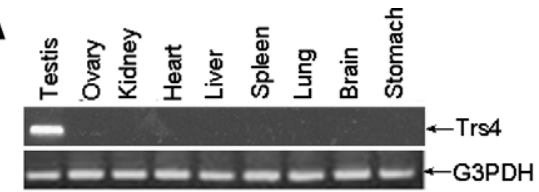

B

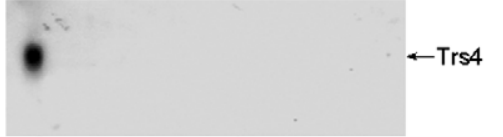

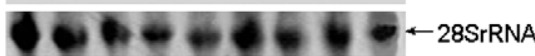

C $\quad$ 는

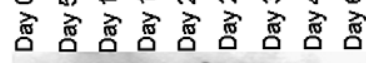
$4 \mathrm{~Kb}-$

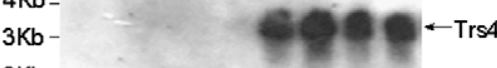

$2 \mathrm{~Kb}-$

$1 \mathrm{~Kb}$

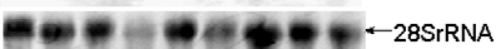

D

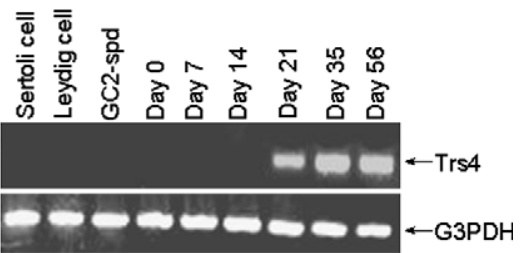

E

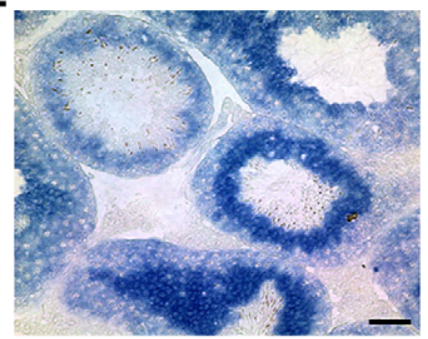

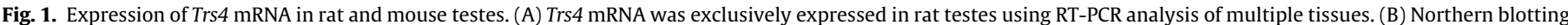

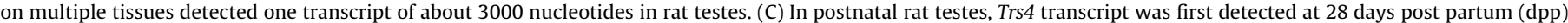

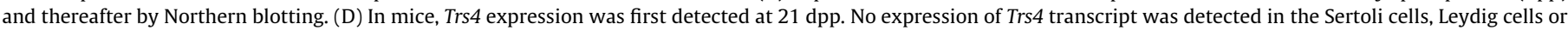

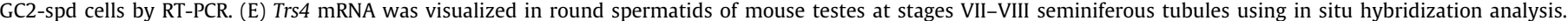
G3PDH RNA and 28S rRNA were used as internal controls for RNA loadings. Scale bars $=50 \mu \mathrm{m}$.

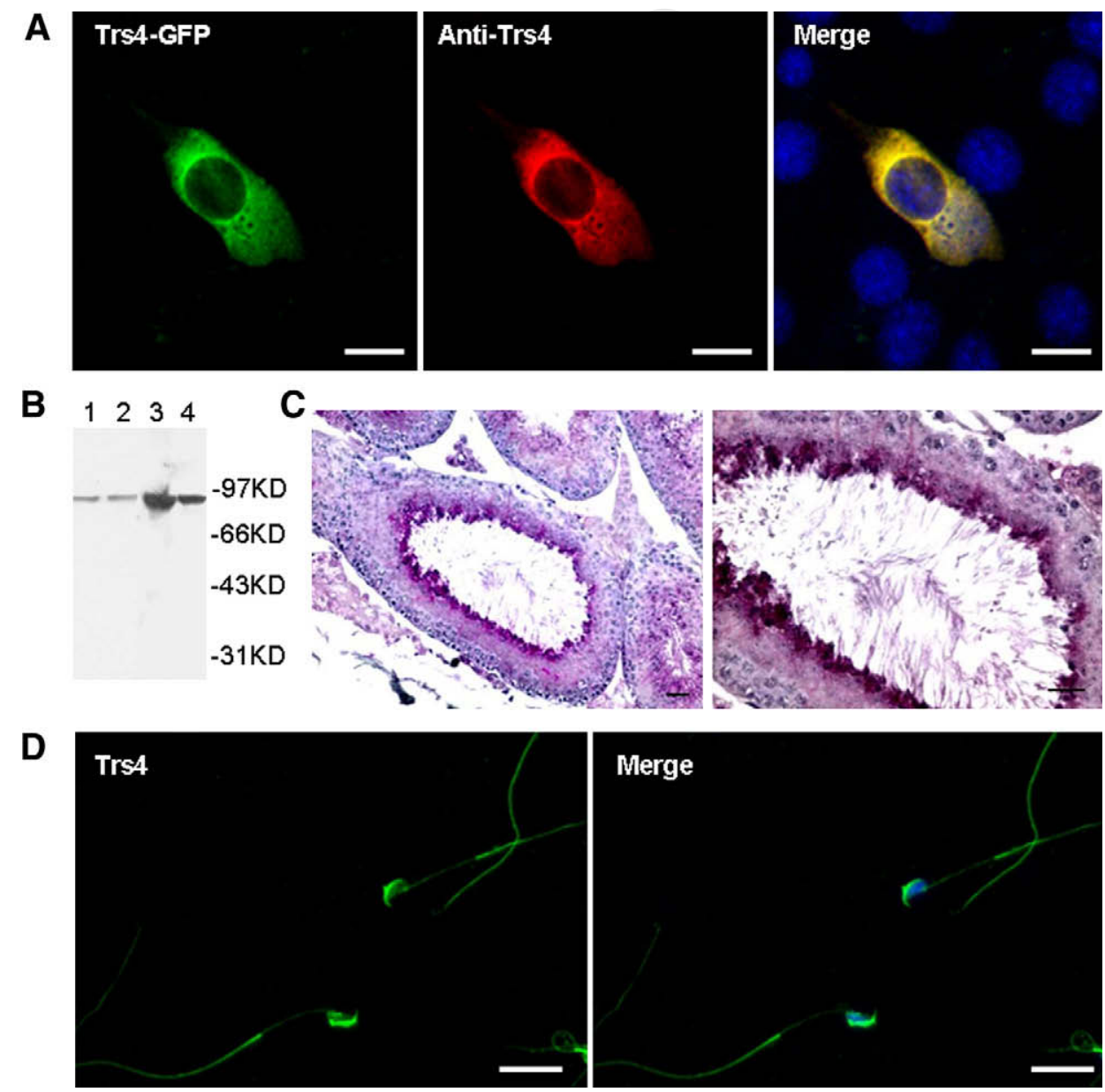

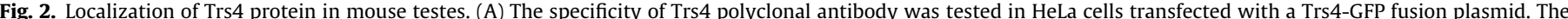

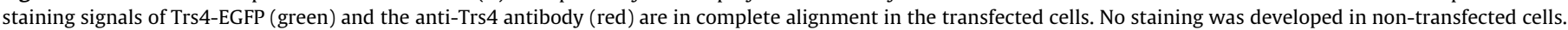

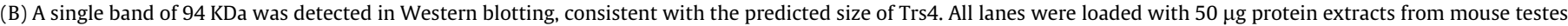

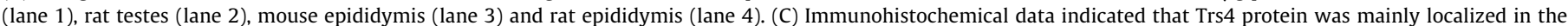

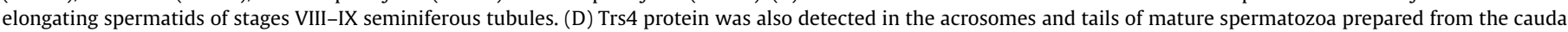

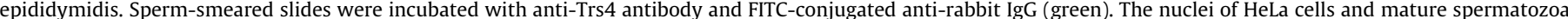

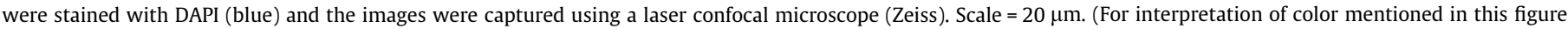
legend the reader is referred to the web version of the article.) 
tion. As shown in Fig. 2A, the GFP-fusion protein was exclusively localized in the cytoplasm of transfected HeLa cells and co-localized with Trs4 immunostaining. Furthermore, no immunostaining signal of Trs4 was detected in untransfected HeLa cells. Specificity of the Trs 4 antibody was also confirmed as a single band of protein with predicted molecular weight of $94 \mathrm{KDa}$ detected in murine total testicular lysates using Western blotting (Fig. 2B). Immunohistochemical data indicated that the Trs4 protein was localized in the elongating spermatids of stages VIII-IX seminiferous tubules (Fig. 2C) and in the acrosomes and tails of mature spermatozoa prepared from the cauda epididymidis (Fig. 2D). A similar localization of Trs4 in rat testes was also observed using immunohistochemistry (data not shown).

\section{Trs4 protein interacts with Rshl-2, Gstmu1, and Ddc8 proteins}

Using a yeast two-hybrid system, putative interacting proteins including Rshl-2, Ddc8, Gstmu1, and Odf-1 were identified using the $\mathrm{T} 2$ bait plasmid of the Trs 4 protein. No interacting protein was identified when T1 was used as a bait. To eliminate potential false positives, in vivo and in vitro binding assays were performed subsequently. In vivo binding assays revealed that the Trs4 protein could coimmunoprecipitate with Rshl-2, Ddc8 and Gstmu1 but not with Odf-1 from the lysates of HEK293T cells transfected with Flag-Trs4 and GFP-tagged interacting proteins (Fig. 3A). The results were further confirmed by the in vitro binding assays, which demonstrated that ${ }^{35}$ S-Met-labeled Myc-Trs4 protein could coimmunoprecipitate with Rshl-2, Ddc8, and Gstmu1 proteins except for Odf-1 (Fig. 3B).

\section{Spatiotemporal expression of Ddc8}

The Ddc8 transcript was uniquely expressed in testes. It was first detected in the mouse testis at $21 \mathrm{dpp}$ and no expression was found in Sertoli cells, Leydig cells or GC2-spd cells (Fig. 4A and $\mathrm{B})$. Ddc8 mRNA was present in round spermatids of the stages VII-VIII seminiferous tubules (Fig. 4C) as revealed by in situ hybridization. The protein was also localized in the tails of elongated spermatids and spermatozoa of stages VII-VIII seminiferous tubules (Fig. 4D) and mature spermatozoa prepared from the cauda epididymidis (Fig. 4E) as detected by immunostaining.

\section{Discussion}

We investigated the expression pattern of the testis-specific gene Trs 4 and its protein interactions with other binding partners. The expression of Trs 4 was examined using RT-PCR, Northern blotting, in situ hybridization and immunostaining techniques. The results of these assays together indicated that Trs 4 is a male germ cell-specific gene whose mRNA is expressed in round spermatids and whose protein is expressed in elongating spermatids and mature spermatozoa. In particular, the protein was mainly located in the acrosomes and tails of mature spermatozoa indicative of its diverse functions during spermiogenesis and/or fertilization. Because the Trs 4 protein sequences are evolutionarily conserved and contain an ubiquitin-like domain and an IQ calmodulin-binding motif, we hypothesize that they might play a role in spermatogenesis through interactions with other proteins.

Using a yeast two-hybrid system, we demonstrated that the Trs4 protein interacted with Rshl-2, Gstmu1, and Ddc8 in the mouse testis. These interactions were further confirmed by in vitro and in vivo binding assays. Rshl-1 and Rshl-2 (radial spokehead-like proteins) are radial spoke proteins that regulate the activity of axonemal inner arm dynein for maintaining the structural integrity and movement of spermatozoa through protein phosphorylation and dephosphorylation [16]. The radial spokes is a T-shaped structure extending from the A-tubule of each outer doublet microtubule to the center of the axoneme of motile structures such as cilia, flagella and the flagella of spermatozoa [17]. In line with this, Trs 4 was localized in the acrosomes and tails of isolated mature epididymal spermatozoa. Therefore, Trs4 protein might be involved in regulating the movement of sperm by interaction with the Rshl-2 protein.

Gstmu1 belongs to the glutathione S-transferase family of enzymes that are responsible for metabolizing a broad range of xenobiotics and carcinogens. Gstmu1 can convert organic compounds to thioethers, a reaction that is the first step in a detoxification process leading to mercapturic acid formation $[18,19]$. Furthermore, Gstmu1 binds to some chemical substances such as steroids, thyroxin, bile acids and bilirubin in a noncatalytic manner to facilitate their transport $[18,20]$. Gstmu 1 modulates stress-mediated signals by repressing apoptosis signal-regulating kinase 1 (ASK1). This activity occurs independently of its catalytic activity in intracellular glutathione metabolism [21]. The expression level of Trs4 decreased in the cryptorchid condition or by treatment of the testes
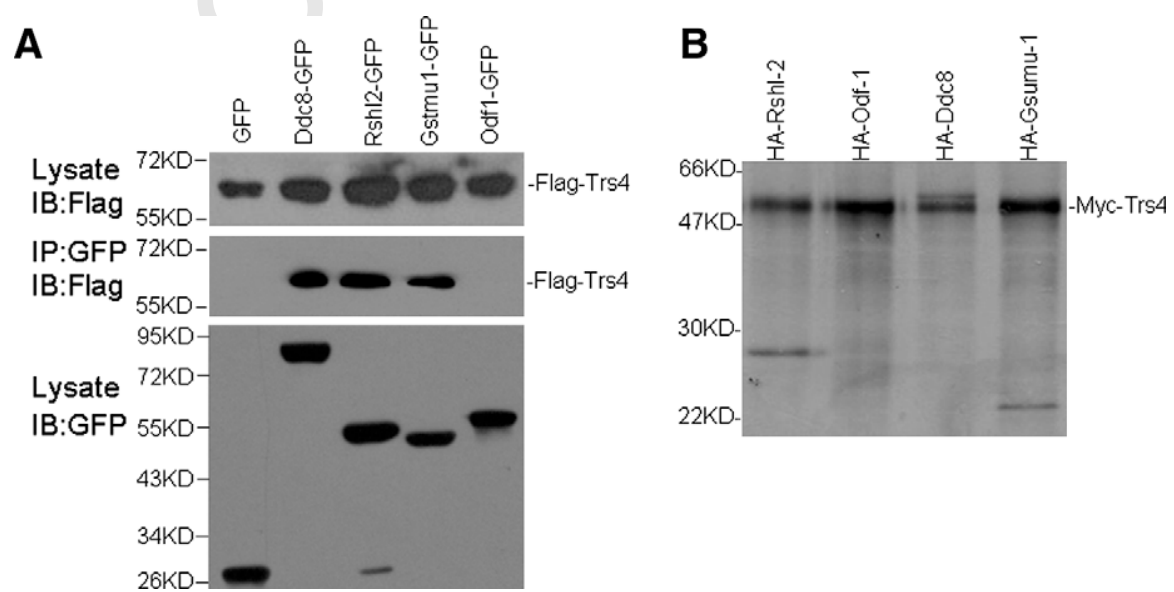

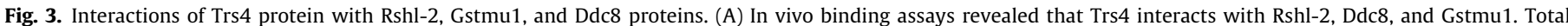

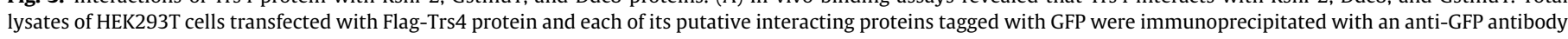

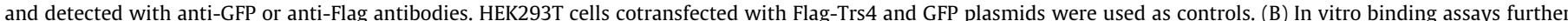

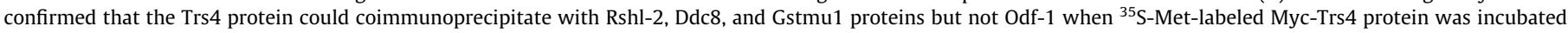
with the interacting proteins. 
A

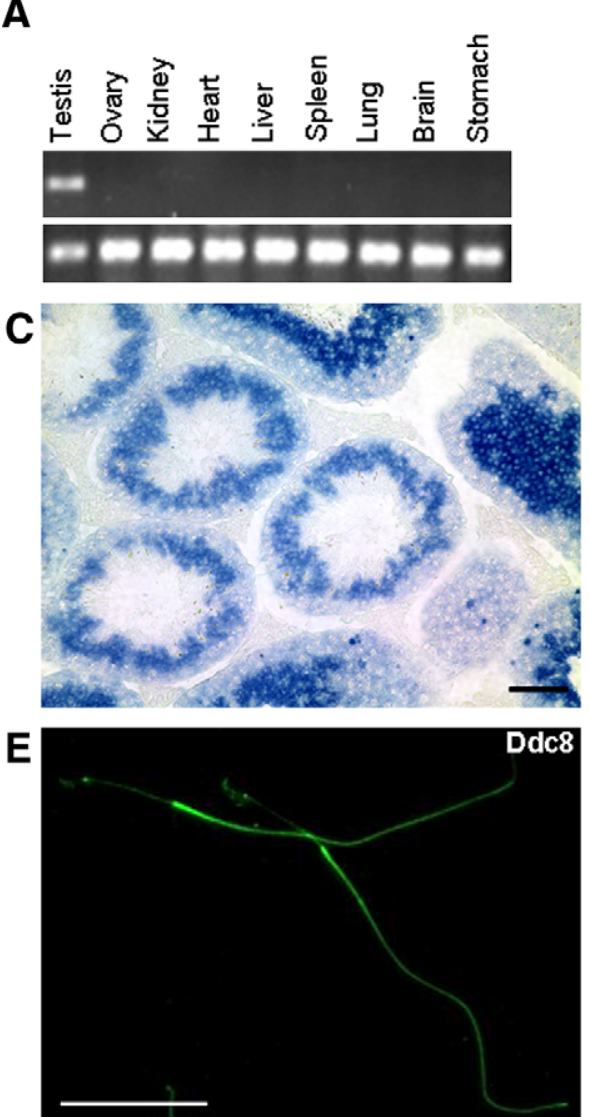

B

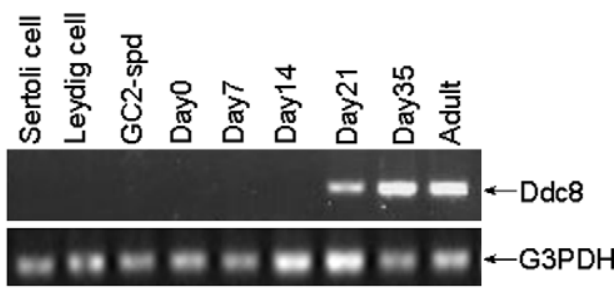

D
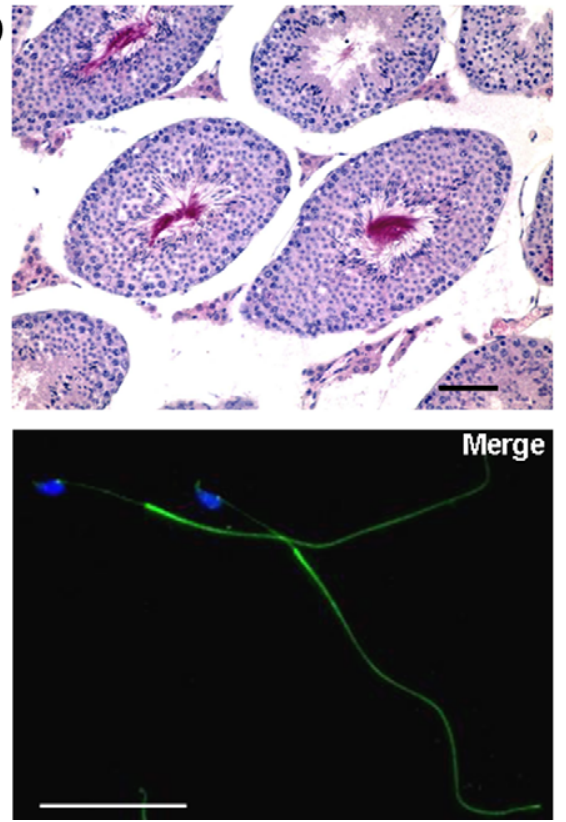

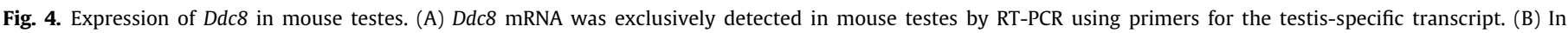

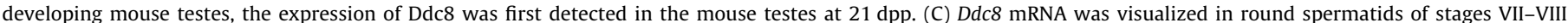

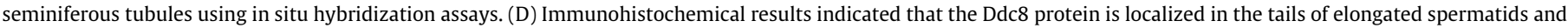

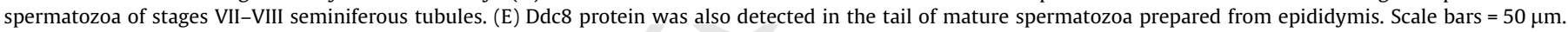

at $43{ }^{\circ} \mathrm{C}$ in a water bath [12]. These treatments increase the level of free radicals in mitochondria that might cause germ cell apoptosis [22]. We hypothesize that Trs4 could participate in regulating the level of oxidative molecules through interaction with Gstmu1. However, this interaction might only facilitate Trs 4 protein transport in the cell.

Ddc8, a newly discovered gene with unknown function, was first identified as a testis-specific gene using differential-display reverse transcription (DDRT)-PCR analysis searching for genes expressed differentially between prepubertal and adult mouse testes [23]. Further studies revealed that $D d c 8$, a gene nested in its host gene family the tissue inhibitors of metalloproteinase 2 (TIMP-2), was not testis-specific. Moreover, Ddc8 expression in non-neural and neural tissues mimicked that of TIMP-2 and was upregulated in response to traumatic brain injury [24]. Interestingly, a specific transcript of Ddc8 consisting of exon-1, exon-2, and exon-3 was found to exist only in mouse testes, while the expression of exon-3 possessed a character of universal expression by RT-PCR and in situ hybridization assays [24]. Using primers specifically designed to detect the testis-specific transcript of $D d c 8$, it was found specifically expressed and regulated in a development-dependent manner in mouse testes. In addition, the mRNA of Ddc 8 was only found in round spermatids and its protein was localized in the tails of elongated spermatids and spermatozoa in stages VII-VII of seminiferous tubules. Ddc 8 and Trs 4 thus share a similar expression pattern in testes and in mature spermatozoa. Moreover, the expression of exon-3 of $D d c 8$ in other tissues including brain, kidney and lung, was also confirmed by RT-PCR (data not shown). Our findings are consistent with data reported previously [24]. We sug- gest that a specific transcript of Ddc8 exists in testes and that its expression is tightly controlled during spermatogenesis.

In conclusion, Trs 4 was specifically expressed in germ cells from early elongating spermatids to mature spermatozoa and it was downregulated by hyperthermia. The Trs4 protein interacted with Rshl-2, Gstmu1, and Ddc8 proteins in mouse testes and co-localized to the tail of mature spermatozoa with Rshl-2 and Ddc8. We suggest that Trs4 alone or in combination with its interacting proteins could play important roles in the processes of spermiogenesis and fertilization.

\section{Acknowledgments}

This study was supported by the National Basic Research Program of China (2006CB944000, 2006CB944004), the National "973" Program (2006CB504001), the NSFC-RGC joint Project (30618005 and N_HKU712/06), Beijing NSF (5073032), the CAS Knowledge Innovation Project (KSCX2-YW-R-5) and the National Natural Science Foundation of China (90508008).

\section{Appendix A. Supplementary data}

Supplementary data associated with this article can be found, in the online version, at doi:10.1016/j.bbrc.2009.08.053.

\section{References}

[1] K.C. Kleene, A possible meiotic function of the peculiar patterns of gene expression in mammalian spermatogenic cells, Mech. Dev. 106 (2001) 3-23. 
[2] N. Schultz, F.K. Hamra, D.L. Garbers, A multitude of genes expressed solely in meiotic or postmeiotic spermatogenic cells offers a myriad of contraceptive targets, Proc. Natl. Acad. Sci. USA 100 (2003) 12201-12206.

[3] P. Sassone-Corsi, Unique chromatin remodeling and transcriptional regulation in spermatogenesis, Science 296 (2002) 2176-2178.

[4] R.I. McLachlan, C. Mallidis, K. Ma, S. Bhasin, D.M. de Kretser, Genetic disorders and spermatogenesis, Reprod. Fertil. Dev. 10 (1998) 97-104.

[5] T. Koji, Y. Hishikawa, Germ cell apoptosis and its molecular trigger in mouse testes, Arch. Histol. Cytol. 66 (2003) 1-16.

[6] R.J. Levine, R.M. Mathew, C.B. Chenault, M.H. Brown, M.E. Hurtt, K.S. Bentley, K.L. Mohr, P.K. Working, Differences in the quality of semen in outdoor workers during summer and winter, N. Engl. J. Med. 323 (1990) $12-16$.

[7] E. Caroppo, C. Niederberger, S. Elhanbly, R. Schoor, L. Ross, G. D'Amato, Effect of cryptorchidism and retractile testes on male factor infertility: a multicenter, retrospective, chart review, Fertil. Steril. 83 (2005) 1581-1584.

[8] J. Guo, S.X. Tao, M. Chen, Y.Q. Shi, Z.Q. Zhang, Y.C. Li, X.S. Zhang, Z.Y. Hu, Y.X. Liu, Heat treatment induces liver receptor homolog-1 expression in monkey and rat sertoli cells, Endocrinology 148 (2007) 1255-1265.

[9] M. Chen, H. Cai, J.L. Yang, C.L. Lu, T. Liu, W. Yang, J. Guo, X.Q. Hu, C.H. Fan, Z.Y. Hu, F. Gao, Y.X. Liu, Effect of heat stress on expression of junction-associated molecules and upstream factors androgen receptor and Wilms' tumor 1 in monkey sertoli cells, Endocrinology 149 (2008) 4871-4882.

[10] X.X. Song, Y.C. Li, Y.Q. Shi, X.Q. Hu, Z.Y. Hu, C.S. Han, Y.X. Liu, Cloning and characterization of a novel spermiogenesis-related gene, T6441, in rat testis, Front. Biosci. 11 (2006) 143-150.

[11] Y.C. Li, X.Q. Hu, K.Y. Zhang, J. Guo, Z.Y. Hu, S.X. Tao, L.J. Xiao, Q.Z. Wang, C.S. Han, Y.X. Liu, Afaf, a novel vesicle membrane protein, is related to acrosome formation in murine testis, FEBS Lett. 580 (2006) 4266-4273.

[12] C.X. Guo, T.S. Tang, X.M. Mu, S.H. Li, G.Q. Fu, H. Liu, Y.X. Liu, Cloning of novel temperature-related expressed sequence tags in rat testis during spermatogenesis, Biochem. Biophys. Res. Commun. 258 (1999) 401-406.

[13] A.F. Karl, M.D. Griswold, Sertoli cells of the testis: preparation of cell cultures and effects of retinoids, Methods Enzymol. 190 (1990) 71-75.
[14] H. Nian, C.H. Fan, S.Y. Liao, Y.Q. Shi, K.Y. Zhang, Y.X. Liu, C.S. Han, RNF151, a testis-specific RING finger protein, interacts with dysbindin, Arch. Biochem. Biophys. 465 (2007) 157-163.

[15] M. Malkov, Y. Fisher, J. Don, Developmental schedule of the postnatal rat testis determined by flow cytometry, Biol. Reprod. 59 (1998) 84-92.

[16] P. Padma, Y. Satouh, K. Wakabayashi, A. Hozumi, Y. Ushimaru, R. Kamiya, K. Inaba, Identification of a novel leucine-rich repeat protein as a component of flagellar radial spoke in the Ascidian Ciona intestinalis, Mol. Biol. Cell 14 (2003) 774-785.

[17] P. Yang, D.R. Diener, C. Yang, T. Kohno, G.J. Pazour, J.M. Dienes, N.S. Agrin, S.M King, W.S. Sale, R. Kamiya, J.L. Rosenbaum, G.B. Witman, Radial spoke proteins of Chlamydomonas flagella, J. Cell. Sci. 119 (2006) 1165-1174.

[18] J. Guo, L. Zimniak, P. Zimniak, J.L. Orchard, S.V. Singh, Cloning and expression of a novel Mu class murine glutathione transferase isoenzyme, Biochem. J. 366 (2002) 817-824.

[19] H. Raza, M.A. Robin, J.K. Fang, N.G. Avadhani, Multiple isoforms of mitochondrial glutathione S-transferases and their differential induction under oxidative stress, Biochem. J. 366 (2002) 45-55.

[20] J.D. Hayes, D.J. Pulford, The glutathione S-transferase supergene family: regulation of GST and the contribution of the isoenzymes to cancer chemoprotection and drug resistance, Crit. Rev. Biochem. Mol. Biol. 30 (1995) 445-600.

[21] S.G. Cho, Y.H. Lee, H.S. Park, K. Ryoo, K.W. Kang, J. Park, S.J. Eom, M.J. Kim, T.S Chang, S.Y. Choi, J. Shim, Y. Kim, M.S. Dong, M.J. Lee, S.G. Kim, H. Ichijo, E.J. Choi, Glutathione S-transferase mu modulates the stress-activated signals by suppressing apoptosis signal-regulating kinase 1, J. Biol. Chem. 276 (2001) 12749-12755.

[22] C.Q. Li, G.N. Wogan, Nitric oxide as a modulator of apoptosis, Cancer Lett. 226 (2005) 1-15.

[23] R.D. Catalano, M. Vlad, R.C. Kennedy, Differential display to identify and isolate novel genes expressed during spermatogenesis, Mol. Hum. Reprod. 3 (1997) 215-221.

[24] D.M. Jaworski, M. Beem-Miller, G. Lluri, R. Barrantes-Reynolds, Potential regulatory relationship between the nested gene DDC8 and its host gene tissue inhibitor of metalloproteinase-2, Physiol. Genomics 28 (2007) 168-178. 